\title{
Dynamically tunable optical bottles from an optical fiber
}

\author{
Chen, Yuhao; Yan, Lu; Rishøj, Lars Søgaard; Steinvurzel, Paul; Ramachandran, Siddharth
}

Published in:

Optics Letters

Publication date:

2012

Document Version

Publisher's PDF, also known as Version of record

Link back to DTU Orbit

Citation (APA):

Chen, Y., Yan, L., Rishøj, L. S., Steinvurzel, P., \& Ramachandran, S. (2012). Dynamically tunable optical bottles from an optical fiber. Optics Letters, 37(16), 3327-3329.

\section{General rights}

Copyright and moral rights for the publications made accessible in the public portal are retained by the authors and/or other copyright owners and it is a condition of accessing publications that users recognise and abide by the legal requirements associated with these rights.

- Users may download and print one copy of any publication from the public portal for the purpose of private study or research.

- You may not further distribute the material or use it for any profit-making activity or commercial gain

- You may freely distribute the URL identifying the publication in the public portal

If you believe that this document breaches copyright please contact us providing details, and we will remove access to the work immediately and investigate your claim. 


\title{
Dynamically tunable optical bottles from an optical fiber
}

\author{
Yuhao Chen, ${ }^{1}$ Lu Yan, ${ }^{1}$ Lars Rishøj, ${ }^{1,2}$ Paul Steinvurzel,,${ }^{1, *}$ and Siddharth Ramachandran ${ }^{1}$ \\ ${ }^{1}$ Boston University, Department of Electrical and Computer Engineering, 8 St. Mary's Street, Boston, Massachusetts 02118, USA \\ ${ }^{2}$ Danish Technical University, Ørsteds Plads 343, 2800 Kongens Lyngby, Denmark \\ *Corresponding author: pes2@bu.edu
}

Received May 10, 2012; revised June 28, 2012; accepted July 3, 2012;

posted July 5, 2012 (Doc. ID 168121); published August 6, 2012

\begin{abstract}
Optical fibers have long been used to impose spatial coherence to shape free-space optical beams. Recent work has shown that one can use higher order fiber modes to create more exotic beam profiles. We experimentally generate optical bottles from Talbot imaging in the coherent superposition of two fiber modes excited with long period gratings, and obtain a $28 \mu \mathrm{m} \times 6 \mu \mathrm{m}$ bottle with controlled contrast up to $10.13 \mathrm{~dB}$. Our geometry allows for phase tuning of one mode with respect to the other, which enables us to dynamically move the bottle in free space. (C) 2012 Optical Society of America

OCIS codes: $140.3300,060.2340,350.4855$.
\end{abstract}

Optical fiber modes are, by definition, spatially coherent states, and so fibers have many applications in beam shaping and spatial filtering. Perhaps the most well-known example of this is in confocal microscopy, where the fiber core both provides a Gaussian spot for diffraction-limited focusing and defines a confocal aperture for light collection [1]. Of course, one is not restricted to using the fundamental mode. Excitation of non-Gaussian core or cladding modes have been shown to be an efficient means of generating hollow beams, beams with phase singularities, and so-called "nondiffracting" Bessel-like beams in free space [2-10].

We recently studied the generation of Bessel-like beams from selective excitation $\mathrm{LP}_{0, m}$ cladding modes and showed a direct correspondence between the radial mode order and effective free-space cone angle of the beam [10]. The spatial superposition of two Bessel-like beams with dissimilar cone angles gives rise to periodically spaced dark foci, also known as optical bottles, along the beam axis, which can be simply understood as interferometric Talbot imaging [11]. Optical bottles have important applications in dipole trapping of low index particles or blue-detuned cold atoms, and in photophoretic trapping [12-14]. One can in principle generate bottles with an arbitrary ensemble of symmetric fiber modes [9], but this allows neither control nor dynamic tunability of the generated bottles.

In this work, we demonstrate optical bottle generation from selective excitation of two Bessel-like $\mathrm{LP}_{0, m}$ fiber modes, a geometry that is directly analogous to doubleaxicon [12] or double annular slit experiments [11]. This is experimentally realized by cascading two long period gratings (LPGs). Our method enables us to independently tune the relative amplitudes and phases of the interference terms, so that we can dynamically control both the depth and location of the bottles. This enables a high degree of control of the beam, available otherwise only from spatial light modulators. Additionally, fibers allow alignment-free remote delivery of the beam.

A schematic of the experiment is shown in Fig. 1(a). We UV write two $1 \mathrm{~cm}$ long gratings, separated by $5 \mathrm{~cm}$, in a high NA single mode fiber (SMBD0980B, OFS). Grating 1 has a pitch $\Lambda=435 \mu \mathrm{m}$ and couples to the $\mathrm{LP}_{0,5}$ mode, whereas grating 2 has $\Lambda=100.6 \mu \mathrm{m}$ and couples to the $\mathrm{LP}_{0,15}$ mode. They are characterized individually by placing index matching gel on one grating and measuring the loss spectrum of the core mode due to the other on an optical spectrum analyzer. The respective spectra are shown in Fig. 1(b). We then cleave the fiber, couple light from an external cavity laser (ECL) (HP 8168F) tuned to $1563 \mathrm{~nm}$, image the end facet on an InGaAs CCD camera (NIR300, Vosskühler GmbH) with a $60 \times$ objective lens, and record a three-dimensional (3-D) image stack as we move the lens and camera longitudinally away from the fiber facet. At the excitation wavelength of $1563 \mathrm{~nm}$, grating 1 couples $28 \%$ of the power into the $\mathrm{LP}_{0,5}$ mode and grating 2 couples $>92 \%(11 \mathrm{~dB})$ of the remaining $\mathrm{LP}_{0,1}$ power to the $\mathrm{LP}_{0,15}$ mode while leaving the $\mathrm{LP}_{0,5}$ mode unaffected, giving a power ratio of $28 \% / 66 \%$. The $y$-cut of the measured tomogram and a two-dimensional (2-D) image of the bottle at $z=216 \mu \mathrm{m}$ are shown in Figs. 1(c) and 1(d).

In Fig. 2(b) we plot the $x$-cut of the tomogram. We measure voids periodically spaced by $97 \mu \mathrm{m}$. The deepest bottle was measured at $z=216 \mu \mathrm{m}$ and has a contrast
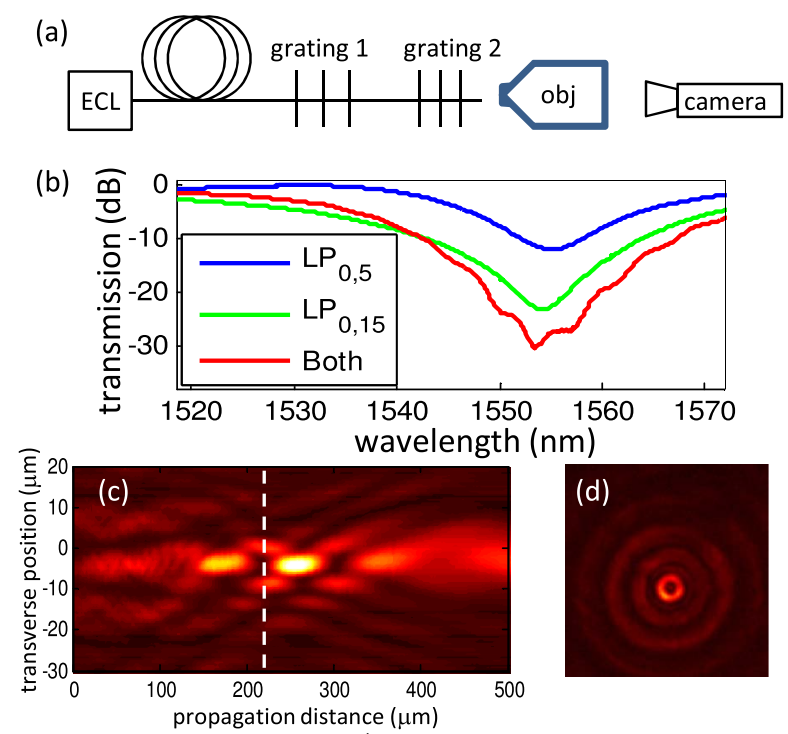

Fig. 1. (Color online) (a) Experimental setup; (b) LPG spectra of grating $1\left(\mathrm{LP}_{0,5}\right)$, grating $2\left(\mathrm{LP}_{0,15}\right)$, and both gratings; (c) a tomogram of the $y$-axis cut; (d) 2-D cross section of the bottle at $z=216 \mu \mathrm{m}$, indicated by the dashed line in (c). An animation of the full 3-D tomogram is shown in Media 1. 
of $10.13 \mathrm{~dB}$ longitudinally and $7.18 \mathrm{~dB}$ transversely, where the contrast is determined by comparing the local minimum with the lower of the two adjacent intensity maxima along the longitudinal and transverse axes respectively. The dimensions of the bottle, defined by a $1 / e$ decrease in local intensity, are $28 \mu \mathrm{m}$ in the longitudinal direction and $6 \mu \mathrm{m}$ in the transverse direction, comparable to the dimensions used to trap microparticles [12]. The associated longitudinal and transverse line plots are shown in Figs. 2(c) and 2(d).

The spacing of the bottles can be understood in terms of the Gouy phase of the individual Bessel-like beams [11]. Though fiber cladding modes are continuous piecewise with $J_{0}, Y_{0}$, and $K_{0}$ dependence, we may approximate the electric field $E$ of these Bessel-like beams arising from these modes [10] as

$$
E(r)=a^{0, m} J_{0}\left(k_{t}^{0, m} r\right) e^{i\left(k_{0} z-\phi^{0, m}(z)+\vartheta^{m}\right)},
$$

where $a^{0, m}$ is the field amplitude of the $\mathrm{LP}_{0, m}$ mode, $k_{t}^{0, m}=k_{0}\left(n_{\mathrm{cl}}^{2}-n_{\mathrm{eff}}^{0, m^{2}}\right)^{1 / 2}$ is the transverse wavenumber of the mode, $k_{0}$ is the free-space wavenumber, $\phi^{0, m}(z)$ is the Gouy phase, $\vartheta^{m}$ is an arbitrary offset phase, $n_{\mathrm{cl}}$ is the cladding index, and $n_{\mathrm{eff}}^{0, m}$ is the effective mode index. We may then write the bottle beam intensity pattern as

$$
\begin{aligned}
I(r, z)= & I^{0, m}(r)+I^{0, n}(r)+2 \sqrt{I^{0, m}(r) I^{0, n}(r)} \\
& \times \cos \left(\phi^{0, m}(z)-\phi^{0, n}(z)+\vartheta^{m, n}\right),
\end{aligned}
$$

where $I^{0, m}(r)$ is the intensity pattern of the $\mathrm{LP}_{0, m}$ mode.

When the mode propagates from the fiber to free-space, $k_{t}^{0, m}$ is preserved, so the square of the longitudinal freespace wavenumber is $\beta^{2}=k_{0}^{2}-k_{t}^{0, m^{2}}$, and therefore [11]

$$
\phi^{0, m}(z)=z\left(k_{0}-\beta\right)=k_{0} z\left(1-\sqrt{\left(1-n_{\mathrm{cl}}^{2}+n_{\mathrm{eff}}^{0, m^{2}}\right)}\right) .
$$

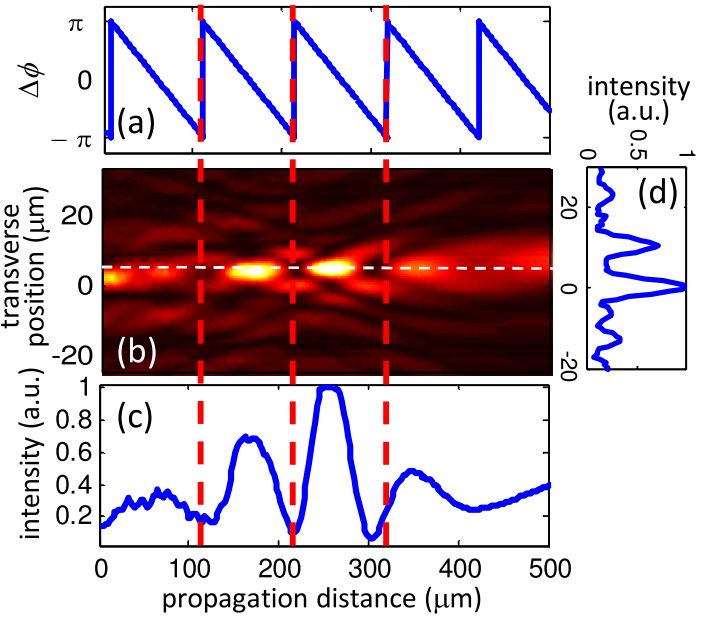

Fig. 2. (Color online) (a) Gouy phase difference between the free space propagation of the $\mathrm{LP}_{0,5}$ and $\mathrm{LP}_{0,15}$ modes as calculated from Eq. (3); (b) tomogram of the $x$-axis cut, where the bottles align with the beat period shown in (a); (c) z-cut of the on-axis beam intensity, indicated by white dashed line in (b); (d) $x$-cut at $z=216 \mu \mathrm{m}$.
In Fig. 2(a) we plot $\phi^{0,5}(z)-\phi^{0,15}(z)$, calculated from the simulated effective indices of the modes. The predicted beat period of $103.19 \mu \mathrm{m}$, indicated by the red dashed lines, matches the measured $97 \mu \mathrm{m}$ spacing between bottles.

Equation (2) shows that the longitudinal size and periodicity of the bottles are determined by $\phi^{0,5}(z)-\phi^{0,15}(z)$, which can be tailored by choosing the appropriate mode orders. We can also dynamically tune the bottles by varying $I^{0, m}, I^{0, n}$, and $\vartheta^{m, n}$. The intensity terms, which relate to power partitioning between the modes, allow us to control the depth of the bottles, whereas the phase term allows us to control their position [15]. We can tune the power partitioning by varying the excitation wavelength; if we operate at a point where one of the LPG spectra has a high slope, tuning the wavelength allows us to vary strength of the mode conversion. We can vary the relative phase by heating the section of fiber between the two gratings [as illustrated in Fig. 4(a)]. In this region, the $\mathrm{LP}_{0, m}$ and $\mathrm{LP}_{0,1}$ modes are copropagating. Since $\mathrm{LP}_{0,1}$ is confined to the Ge-doped core and $\mathrm{LP}_{0, m}$ is primarily confined to the pure silica cladding, these modes have a differential thermo-optic response, and the accumulated phase difference can be written as

$$
\Delta \phi(T)=\left(d n_{\mathrm{eff}}^{0,1} / d T-d n_{\mathrm{eff}}^{0, m} / d T\right) k_{0} L \Delta T,
$$

where $L$ is the length of the heated section and $\Delta T$ is the temperature difference. We experimentally demonstrate both tuning mechanisms.

In Fig. (3), we show the effect of wavelength tuning on the power partitioning as indicated on the individual contour plots [also cf. grating spectra in Fig. 1(b)]. Changing the wavelength primarily changes the $\mathrm{L} \overline{\mathrm{P}}_{0,5}$ conversion efficiency, since grating 2 is relatively strong over the $1540-1551 \mathrm{~nm}$ range, and allows us to change the onaxis contrast from 8.74 to $2.68 \mathrm{~dB}$. We note that we obtain
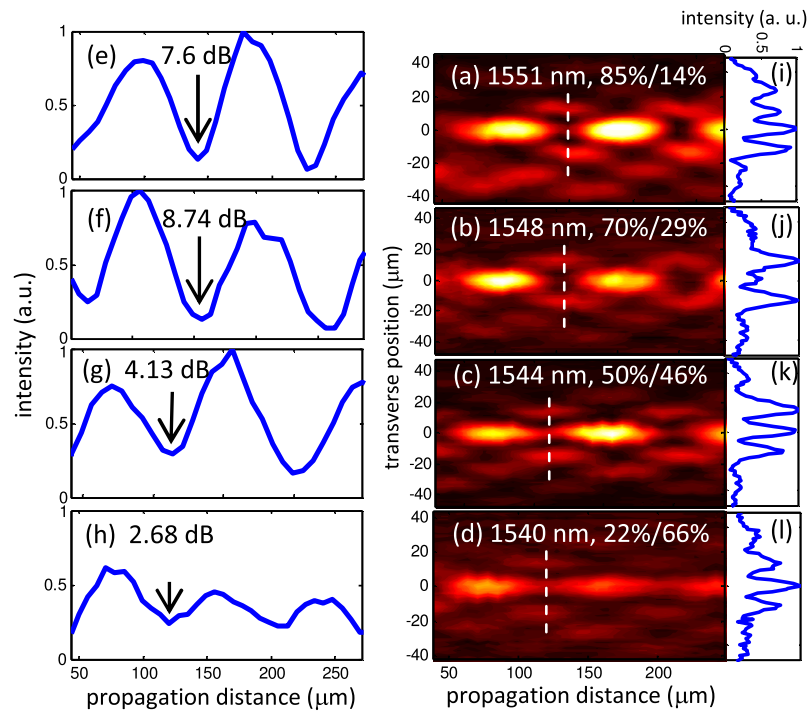

Fig. 3. (Color online) Tuning the bottle contrast by wavelength tuning. (a)-(d) Tomograms at the indicated wavelengths and relative power in $\mathrm{LP}_{0,5} / \mathrm{LP}_{0,15} ;(\mathrm{e})-(\mathrm{g})$ their respective on-axis intensity lineplots; (i)-(l) their respective transverse intensity plots along the white dashed lines. 

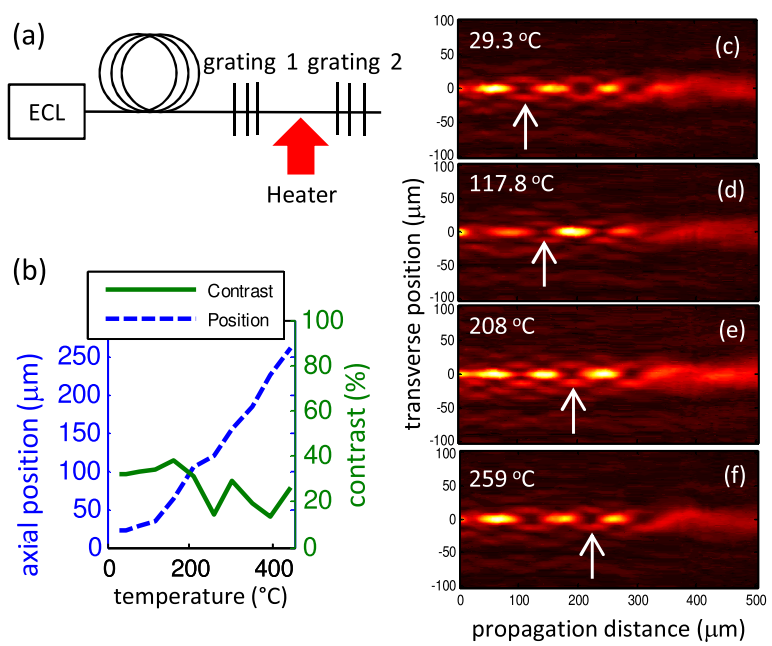

Fig. 4. (Color online) (a) Schematic diagram showing heat applied between the two gratings; (b) axial position of a dark focus as a function of temperature (blue dashed curve) and contrast of this focus as it moves (green solid curve); (c)-(f) tomograms at different measured temperatures, where the white arrows indicate the dark focus tracked in (b). An animation of the tomogram evolution as a function temperature is shown in Media 2.

the best contrast for $70 \%$ and $29 \%$ of $\mathrm{LP}_{0,5}$ and $\mathrm{LP}_{0,15}$, respectively, not at $50 \% / 46 \%$ partitioning, since the contrast depends on local intensity in the transverse plane, and not the total power.

For the phase tuning experiment, we positioned the fiber section between the two gratings in a $3 \mathrm{~cm}$ long ceramic tube coiled with a $\mathrm{NiCr}$ wire resistive heater. Fig. 4(b) shows the position of one of the bottles as we increase the temperature. The dark focus moved a total distance of $241 \mu \mathrm{m}$ as the temperature was varied by $420{ }^{\circ} \mathrm{C}$. Effectively, this varies the $\vartheta^{m, n}$ term in Eq. (2), allowing us to control the axial position of the dark focus. The green line in Fig. 4(b) shows that the contrast of this focus does change as it moves, due to the fact that onaxis intensities of the interfering modes are not in fact constant [10], though this can be mitigated by waveguide design. However, the contrast is never worse than $4 \mathrm{~dB}$. Our results also allow us to estimate the differential thermo-optic coefficients of the core and cladding modes. Since moving the bottle by one period of the bottle spacing corresponds to a $2 \pi$ phase shift, we can estimate that we experimentally generate a phase shift of $0.027 \mathrm{rad} /{ }^{\circ} \mathrm{C}$. This means that the differential thermooptic coefficient of the $\mathrm{LP}_{0,5}$ and $\mathrm{LP}_{0,1}$ modes is $2.2 \times$ $10^{-7}{ }^{\circ} \mathrm{C}^{-1}$, which, based on modal simulations, allows us to estimate the differential thermo-optic coefficient of the core and cladding materials as $4.3 \times 10^{-7}{ }^{\circ} \mathrm{C}^{-1}$. This experiment thus also demonstrates a path toward fiber characterization.

Finally, we note that we are also able to create a $20 \mu \mathrm{m} \times 8 \mu \mathrm{m}$ bottle with a $7.16 \mathrm{~dB}$ contrast using a single grating device based on interference between $\mathrm{LP}_{0,1}$ and $\mathrm{LP}_{0,15}$. We obtain optimal contrast for $50 \%$ power partitioning between the two modes. Since the Gaussian $\mathrm{LP}_{0,1}$ mode strongly diffracts, a description of the bottle formation requires some obvious modifications to Eq. (2), but we can understand that this process works primarily because the intensity of $\mathrm{LP}_{0,1}$ is very high. The local on-axis intensity of $\mathrm{LP}_{0,1}$ far from the fiber facet can still be comparable to the Bessel-like mode and give high extinction, though we get just one deep bottle.

In summary, we have demonstrated the generation of a $28 \mu \mathrm{m} \times 6 \mu \mathrm{m}$ dark focus from an optical fiber. We have also demonstrated the ability of depth tuning via wavelength control and longitudinal position tuning via temperature control. This shows the versatility of this fiber technique. The ease of mode order choice, the ability to control relative phase between the modes, and the fact that this is based on a mature fiber grating fabrication technology indicate that this technique would be attractive both as a device for generating tunable bottle beams and for applications that require remote delivery of such beams.

We thank N. Božinović for his help with the imaging setup. This work was done with the support of the Office of Naval Research, grant nos. N00014-11-1-0133 and N00014-11-1-0098.

\section{References}

1. K. P. Ghiggino, M. R. Harris, and P. G. Spizzirri, Rev. Sci. Instrum. 63, 2999 (1992).

2. K. Oh, S. Choi, Y. Jung, and J. W. Lee, J. Lightwave Technol. 23, 524 (2005).

3. M.-L. Hu, C.-Y. Wang, Y.-J. Song, Y.-F. Li, L. Chai, E. E. Serebryannikov, and A. M. Zheltikov, Opt. Express 14, 4128 (2006).

4. V. Pureur, J. C. Knight, and B. T. Kuhlmey, Opt. Express 18, 8906 (2010).

5. T. Grosjean, D. Courjon, and M. Spajer, Opt. Commun. 203, 1 (2002).

6. A. Witkowska, S. G. Leon-Saval, A. Pham, and T. A. Birks, Opt. Lett. 33, 306 (2008).

7. S. Ramachandran, P. Kristensen, and M. F. Yan, Opt. Lett. 34, 2525 (2009).

8. N. Božinović, S. Golowich, P. Kristensen, and S. Ramachandran, Opt. Lett. 37, 2451 (2012).

9. X. Zhu, A. Schülzgen, L. Li, and N. Peyghambarian, Appl. Phys. Lett. 94, 201102 (2009).

10. P. Steinvurzel, K. Tantiwanichapan, M. Goto, and S. Ramachandran, Opt. Lett. 36, 4671 (2011).

11. S. Chávez-Cerda, E. Tepichin, M. A. Meneses-Nava, G. Ramirez, and J. M. Hickmann, Opt. Express 3, 524 (1998).

12. B. P. S. Ahluwalia, W. C. Cheong, X.-C. Yuan, L.-S. Zhang, S.-H. Tao, J. Bu, and H. Wang, Opt. Lett. 31, 987 (2006).

13. L. Isenhower, W. Williams, A. Dally, and M. Saffman, Opt. Lett. 34, 1159 (2009).

14. V. G. Shvedov, A. S. Desyatnikov, A. V. Rode, W. Krolikowski, and Y. S. Kivshar, Opt. Express 17, 5743 (2009).

15. T. Čižmár, M. Šiler, and P. Zemánek, Appl Phys. B 84, 197 (2006). 